\author{
Series A
}

I. MATHEMATICA

436

\title{
EXTENSION OVER QUASICONFORMALLY EQUIVALENT CURVES
}

BY

SEPPO RICKMAN

H E L S I N K I 1969

S U O M A L A I N N T I E D E A K A T E M A

doi:10.5186/aasfm.1969.436 
Communicated 8 November 1968 by Olli Lehto and K. I. Virtanen 


\section{Introduction}

Two Jordan curves $\gamma$ and $\gamma^{\prime}$ lying in the extended complex plane $\mathbf{C}^{*}=\mathbf{C} \cup\{\infty\}$ are called quasiconformally equivalent if there exists a quasiconformal mapping of $\mathbf{C}^{*}$ onto itself which maps $\gamma$ onto $\gamma^{\prime}$. In [5] there is given a metrical condition which characterizes this equivalence relation in terms of the existence of homeomorphisms $\varphi: \gamma \rightarrow \gamma^{\prime}$ which can be extended to quasiconformal mappings of $\mathbf{C}^{*}$. Those Jordan curves which are equivalent with circles, called quasiconformal curves or quasicircles, form a special equivalence class denoted here by $\Gamma_{0}$. The class $\Gamma_{0}$ can also be described to consist exactly of those Jordan curves which permit a quasiconformal reflection ([2]).

Let $G$ and $G^{\prime}$ be Jordan domains. We denote by $E\left(G, G^{\prime}\right)$ the family of quasiconformal mappings $f: G \rightarrow G^{\prime}$ which can be quasiconformally extended to $\mathbf{C}^{*}$. By Satz II.8.1 in [3] it follows that if a quasiconformal mapping $f: G \rightarrow G^{\prime}$ has a quasiconformal extension to a domain which contains the closure $\bar{G}$, then $f \in E\left(G, G^{\prime}\right)$. Quasiconformal curves have the following property with respect to quasiconformal extension: If the boundaries $\partial G$ and $\partial G^{\prime}$ belong to $\Gamma_{0}$, then every quasiconformal mapping $f: G \rightarrow G^{\prime}$ belongs to $E\left(G, G^{\prime}\right)$. This result is an immediate consequence of the definition of $\Gamma_{0}$.

In this paper we study to which extent the extension property of $\Gamma_{0}$ is valid for the other equivalence classes. Especially we ask whether there is some equivalence class $\Gamma$ different from $\Gamma_{0}$ such that every quasiconformal mapping $f: G \rightarrow G^{\prime}$, where $G$ and $G^{\prime}$ are Jordan domains with boundaries in $\Gamma$, belongs to $E\left(G, G^{\prime}\right)$. That no such class exists is established in Theorem 2. It is also natural to ask how small $E\left(G, G^{\prime}\right)$ can be. Theorem 3 gives an answer in this direction and shows that there is even an equivalence class $\Gamma$ such that all mappings in $E\left(G, G^{\prime}\right)$, where the boundaries $\partial G$ and $\partial G^{\prime}$ are in $\Gamma$, coincide on the boundary, that is, $E\left(G, G^{\prime}\right)$ is smallest possible according to Corollary 1 of Theorem 1.

Research supported in part by the Air Force Office of Scientific Research, Contract AF 49 (638) 1591. 


\section{Extension properties}

We first restate Theorem 3 in [5] in a special case which is more appropriate for our present purposes:

Theorem 1. Let $f: \mathbf{C}^{*} \rightarrow \mathbf{C}^{*}$ be a homeomorphism which is quasiconformal off a Jordan curve $\gamma$. If there exists a quasiconformal mapping $g: \mathbf{C}^{*} \rightarrow \mathbf{C}^{*}$ such that $g|\gamma=f| \gamma$, then $f$ is quasiconformal.

Corollary 1. Let $f_{1}$ and $f_{2}$ be quasiconformal mappings of a Jordan domain $G$ onto a Jordan domain $G^{\prime}$ such that they coincide on the boundary $\partial G$. Then $f_{1} \in E\left(G, G^{\prime}\right)$ if and only if $f_{2} \in E\left(G, G^{\prime}\right)$.

Corollary 2. Assume $f \in E\left(G, G^{\prime}\right)$ where $G$ and $G^{\prime}$ are Jordan domains. If $g: \mathbf{C}^{*} \rightarrow \mathbf{C}^{*}$ is a homeomorphic extension of $f$ which is quasiconformal in $\mathbf{C}^{*}-\bar{G}$, then $g$ is a quasiconformal extension of $f$.

We need some notation. The complement and the euclidean diameter of a set $A$ are denoted by $C_{A}$ and $d(A)$ respectively. We denote by $B_{r}(z)$ the open disc and by $S_{r}(z)$ the circle with radius $r$ and center $z$. By $z_{1} z_{2}$ we mean the open line segment with endpoints $z_{1}$ and $z_{2}$. If $\alpha$ is a Jordan arc and $w_{1}, w_{2} \in \alpha$, then $\alpha\left(w_{1}, w_{2}\right)$ is the open subare of $\alpha$ with endpoints $w_{1}$ and $w_{2}$. The absolute value of the cross-ratio of the sequence $z_{1}, z_{2}, z_{3}, z_{4}$ is denoted by $\left|z_{1}, z_{2}, z_{3}, z_{4}\right|$. For finite distinct points we have

$$
\left|z_{1}, z_{2}, z_{3}, z_{4}\right|=\frac{\left|z_{1}-z_{3}\right|\left|z_{2}-z_{4}\right|}{\left|z_{1}-z_{4}\right|\left|z_{2}-z_{3}\right|} .
$$

By $\mu(r), 0<r<1$, we denote the modulus of the unit disc slit along the real axis from 0 to $r$.

The following result ([1]) is a consequence of a distortion theorem of Teichmüller:

Lemma 1. If $f: \mathbf{C}^{*} \rightarrow \mathbf{C}^{*}$ is a $K$-quasiconformal mapping, then

$$
\left|f\left(z_{1}\right), \ldots, f\left(z_{4}\right)\right| \leq L\left(K,\left|z_{1}, \ldots, z_{4}\right|\right)
$$

holds for distinct points $z_{1}, \ldots, z_{4} \in \mathbf{C}^{*}$, where we have used the notation

$$
L(K, t)=\frac{1}{\left(\mu^{-1}\left(K \mu\left((1+t)^{-1 / 2}\right)\right)\right)^{2}}-1 .
$$

The fact that the class $\Gamma_{0}$ of quasiconformal curves is the only class which has the special extension property mentioned earlier is stated as follows.

Theorem 2. Let $G$ and $G^{\prime}$ be Jordan domains. Then every quasiconformal mapping $f: G \rightarrow G^{\prime}$ has a quasiconformal extension to $\mathbf{C}^{*}$ if and only if $\partial G$ and $\partial G^{\prime}$ are quasiconformal curves. Moreover, if $\partial G$ is not 
quasiconformal, then for every $K>1$ there exists a $K$-quasiconformal mapping $f: G \rightarrow G^{\prime}$ which cannot be extended quasiconformally over $\partial G$.

Proof. We already pointed out that if $\gamma=\partial G$ and $\gamma^{\prime}=\partial G^{\prime}$ are quasiconformal curves, then every quasiconformal mapping $f: G \rightarrow G^{\prime}$ belongs to $E\left(G, G^{\prime}\right)$. Since the inverse of a $K$-quasiconformal mapping is $K$-quasiconformal, it suffices to prove the last part of the theorem.

Let $K$ be greater than 1 . We can assume that $\infty \in \gamma \cap \gamma^{\prime}$. Suppose that $\gamma$ is not quasiconformal. If $\gamma^{\prime}$ is quasiconformal, the family $E\left(G, G^{\prime}\right)$ is empty. Suppose therefore that $\gamma^{\prime}$ is not quasiconformal at a point $z^{\prime}$ ([4]). If now $\gamma$ is quasiconformal at a point $z$, the conformal mapping of $G$ onto $G^{\prime}$ whose extension to the boundary maps $z$ onto $z^{\prime}$ has no quasiconformal extension to $\mathbf{C}^{*}$. Consequently, we can assume that $\gamma$ is not quasiconformal at any point.

Let now $Q=G\left(z_{1}, z_{2}, z_{3}, \infty\right)$ and $Q^{\prime}=G^{\prime}\left(z_{1}{ }^{\prime}, z_{2}{ }^{\prime}, z_{3}{ }^{\prime}, \infty\right)$ be quadrilaterals with modulus 1 , and let $g$ and $g^{\prime}$ be the canonical mappings of $Q$ and $Q^{\prime}$ respectively onto the square $A=A(0,1,1+i, i)$. Using the same notation for the extension of $g$ to the closure $\bar{Q}$ we denote $w_{n}=$ $g^{-1}\left(2^{-2 n+1}\right), n=1,2, \ldots$. Choose $\delta$ such that $0<\delta<1$ and $1 /(1-\delta)$ $<\sqrt{K}$.

Let $\gamma_{n, 1}, \gamma_{n, 2}, \ldots$ be a sequence of open subares of $\gamma-\{\infty\}$ such that $\gamma_{n, m} \ni w_{n}, m=1,2, \ldots$, and $\lim _{m \rightarrow \infty} d\left(\gamma_{n, m}\right)=0$. Since $\gamma$ is not quasiconformal at $w_{n}$, there exist for every $m$ successive points $a_{n, m}^{1}$, $a_{n, m}^{2}, a_{n, m}^{3}$ of $\gamma_{n, m}$ such that

$$
\frac{\left|a_{n, m}^{1}-a_{n, m}^{2}\right|}{\left|a_{n, m}^{1}-a_{n, m}^{3}\right|}>m .
$$

Denote by $b_{n, m}^{j}$ the first point of $\overline{\gamma_{n, m}\left(a_{n, m}^{j}, a_{n, m}^{2}\right)}$ from $a_{n, m}^{2}$ which belongs to the line segment $\overline{a_{n, m}^{1} a_{n, m}^{3}}, j=1,3$. Let $H_{n, m}$ be the bounded Jordan domain whose boundary is $\overline{b_{n, m}^{1} b_{n, m}^{3}} \cup \gamma_{n, m}\left(b_{n, m}^{1}, b_{n, m}^{3}\right)$ and let $U_{n, m}$ be a circular neighborhood of $a_{n, m}^{2}$ such that $U_{n, m} \cap\left(\left(\gamma-\gamma_{n, m}\left(b_{n, m}^{1}, b_{n, m}^{3}\right)\right) \cup\right.$ $b_{n, m}^{1}\left(b_{n, m}^{3}\right)=\varnothing$. Then for every $n$ at least one of the following cases occur:

$$
\begin{aligned}
& G \cap U_{n, m} \subset H_{n, m} \text { for infinitely many } m, \\
& C_{\bar{G}} \cap U_{n, m} \subset H_{n, m} \text { for infinitely many } m .
\end{aligned}
$$

Let $E_{n}$ be a disc such that $E_{n} \subset G^{\prime}$ if (a) occurs for $n$ and $E_{n} \subset C_{\bar{G}^{\prime}}$ otherwise, and such that $\partial E_{n}$ contains a point $a_{n}^{\prime}$ of $g^{\prime-1}\left(\omega_{n}\right)$ where $\omega_{n}$ is the interval

$$
\left(\frac{1}{2^{2 n-1}}-\frac{\delta}{2^{2 n}}, \frac{1}{2^{2 n-1}}+\frac{\delta}{2^{2 n}}\right)
$$


of the real axis. Now denote

$$
\sigma_{n}=L\left(n, \frac{\left|z_{1}^{\prime}-a_{n}^{\prime}\right|}{\left|\zeta_{n}^{\prime}-a_{n}^{\prime}\right|}\right)
$$

where $L$ is defined by (2.1) and where $\zeta_{n}^{\prime}$ is the center of $E_{n}$.

For every $n$ we choose $m_{n}>n$ such that $\gamma_{n, m_{n}}$ is contained in a disc $D_{n}$ for which

$$
\frac{\left|u-u^{*}\right|}{\left|u-z_{1}\right|}<\frac{1}{\sigma_{n}}
$$

if $u, u^{*} \in D_{n}$, such that $g\left(\gamma_{n, m_{n}}\right) \subset \omega_{n}$, and that $G \cap U_{n, m_{n}} \subset H_{n, m_{n}}$ if (a) holds for $n$ and $C_{\bar{G}} \cap U_{n, m_{n}} \subset H_{n, m_{n}}$ otherwise. For simplicity we write $a_{n}^{j}=a_{n, m_{n}}^{j}, b_{n}^{j}=b_{n, m_{n}}^{j}, j=1,2,3, H_{n}=H_{n, m_{n}}$, and $U_{n}=U_{n, m_{n}}$.

We define now subsets of $A$ for every $n, n=1,2, \ldots$, as follows:

$$
\begin{aligned}
& V_{n}=\left\{z \in A\left|\frac{1}{2^{2 n}}<\operatorname{Re} z<\frac{1}{2^{2 n-1}}\right|,\right. \\
& W_{n}=\left\{z \in A \mid \frac{1}{2^{2 n-1}}<\operatorname{Re} z<\frac{1}{2^{2 n-2}}\right\}, \\
& X_{n}=\left\{z \in A \mid \frac{1}{2^{2 n}}<\operatorname{Re} z<v_{n}\right\}, \\
& Y_{n}=\left\{z \in A\left|v_{n}<\operatorname{Re} z<\frac{1}{2^{2 n-2}}\right|,\right.
\end{aligned}
$$

where $\quad v_{n}=g\left(a_{n}^{2}\right)$. Let $h: A \rightarrow A$ be a homeomorphism for which the restrictions $h \mid V_{n}$ and $h \mid W_{n}$ are the natural affine mappings onto $X_{n}$ and $Y_{n}$ respectively, $n=1,2, \ldots$. A homeomorphism $h^{\prime}: A \rightarrow A$ is defined similarly by the use of the numbers $v_{n}^{\prime}=g^{\prime}\left(a_{n}^{\prime}\right)$.

According to our choice of $\delta$, the mapping $f=g^{\prime-1} \circ h^{\prime} \circ h^{-1} \circ g$ of $G$ onto $G^{\prime}$ is $K$-quasiconformal. We claim that $f$ has no quasiconformal extension over $\gamma$. Suppose such an extension exists, i.e. there is a $K_{0^{-}}$ quasiconformal mapping $F: \mathbf{C}^{*} \rightarrow \mathbf{C}^{*}$ for some $K_{0}<\infty$ such that $F \mid G=f$. Assume $n>K_{0}$. If we apply Lemma 1 to the mapping $F^{-1}$ and the sequence $z_{1}^{\prime}, \zeta_{n}^{\prime}, a_{n}^{\prime}, \infty$, we obtain from (2.2) that $F\left(D_{n}\right)$ and hence $F\left(H_{n}\right)$ do not contain the point $\zeta_{n}^{\prime}$. Let $T_{n}$ be the circular annulus with boundary components $S_{r_{n}}\left(b_{n}^{1}\right)$ and $S_{t_{n}}\left(b_{n}^{1}\right)$ where $r_{n}=\left|b_{n}^{1}-b_{n}^{3}\right|$ and $t_{n}=\left|b_{n}^{1}-a_{n}^{2}\right|$. Suppose (a) holds for $n$. Then $E_{n} \subset G^{\prime}$ and hence $l_{n}=F^{-1}\left(a_{n}^{\prime} \zeta_{n}^{\prime}\right) \subset G$. Let $c_{n}$ be a point of $U_{n} \cap l_{n}$. Since $c_{n} \in H_{n}$ and $F^{-1}\left(\zeta_{n}^{\prime}\right) \in C_{H_{n}}$, there is a point $d_{n} \in l_{n} \cap \partial H_{n}$. But this is possible only if $d_{n} \in b_{n}^{1} b_{n}^{3}$ and there must therefore be a point $e_{n} \in l_{n} \cap S_{r_{n}}\left(b_{n}^{1}\right)$. The ring 
$T_{n}^{\prime}=F\left(T_{n}\right)$ separates the points $F\left(b_{n}^{1}\right)$ and $F\left(e_{n}\right)$ from the points $a_{n}^{\prime}$ and $\infty$. Since $\left|F\left(b_{n}^{1}\right)-F\left(e_{n}\right)\right| \geq\left|a_{n}^{\prime}-F\left(e_{n}\right)\right|$, we get by the modulus theorem of Teichmüller ([3], p. 58) the following estimate

$$
M\left(T_{n}^{\prime}\right) \leq 2 \mu\left(\frac{1}{\sqrt{2}}\right)=\pi .
$$

The case that (a) does not hold for $n$ is treated similarly. But $\lim M\left(T_{n}\right)$ $=\infty$ because

$$
\frac{\left|b_{n}^{1}-a_{n}^{2}\right|}{\left|b_{n}^{1}-b_{n}^{3}\right|}>m_{n}-1
$$

and $m_{n}>n, n=1,2, \ldots$, and we have a contradiction with the quasiconformality of $F$. The theorem is proved.

Remark. By a similar but more complicated argument one can actually show that the statement in Theorem 2 holds also for $K=1$.

\section{An example}

Let $\gamma$ and $\gamma^{\prime}$ be quasiconformally equivalent and let $g: \mathbf{C}^{*} \rightarrow \mathbf{C}^{*}$ be a quasiconformal mapping such that $g(\gamma)=\gamma^{\prime}$. Let $G$ be one of the complementary domains of $\gamma$ and denote $G^{\prime}=g(G)$. Then $f_{0}=g \mid G$ $\in E\left(G, G^{\prime}\right)$, and Corollary 1 of Theorem 1 says that every quasiconformal mapping $f: G \rightarrow G^{\prime}$ which coincides with $f_{0}$ on the boundary belongs to $E\left(G, G^{\prime}\right)$. Note that if Jordan domains $D$ and $D^{\prime}$ with $\partial D=\gamma$ and $\partial D^{\prime}=\gamma^{\prime}$ are chosen so that $g(D) \neq D^{\prime}$, then $E\left(D, D^{\prime}\right)$ may be empty. We shall now establish an example which gives the following result.

Theorem 3. There is an equivalence class $\Gamma$ such that if $G$ and $G^{\prime}$

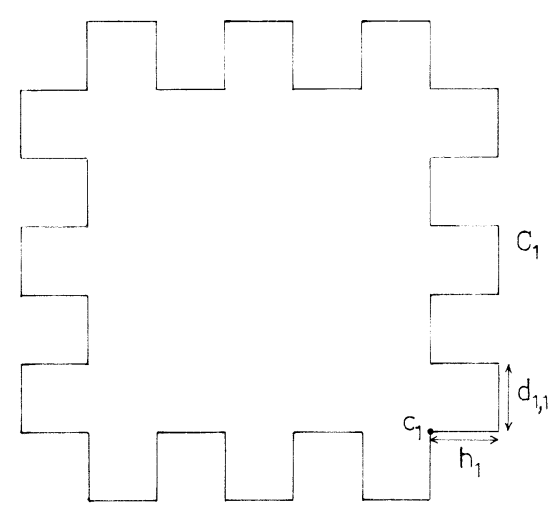

Figure 1. 
are Jordan domains such that $\partial G, \partial G^{\prime} \in \Gamma$, then all mappings in $E\left(G, G^{\prime}\right)$ coincide on the boundary.

This follows immediately from the following theorem.

Theorem 4. There is a Jordan curve $\gamma$ such that if $f: \mathbf{C}^{*} \rightarrow \mathbf{C}^{*}$ is a quasiconformal mapping which maps $\gamma$ onto itself, then $f \mid \gamma$ is the identity mapping.

Proof. To construct a Jordan curve with the required property we start with the segmental Jordan curve $C_{1}$ and a point $c_{1}$ on $C_{1}$ as shown in Figure 1 where $d_{1,1}=h_{1}=1 / 5$. We shall form a sequence $C_{1}, C_{2}, \ldots$ of segmental Jordan curves inductively as follows. Suppose $C_{l-1}$ is constructed and the point $c_{l-1}$ on $C_{l-1}$ chosen. To construct $C_{l}$ we deform, starting from the point $c_{l-1}$, the line segment parts of $C_{l-1}$ into successive wavelike closed arcs $C_{l, 1}, \ldots, C_{l, n}$ as shown in Figure 2 (which is an illustration of the case where $l$ is even) such that the height and the width of the waves satisfy certain conditions stated below. We set

$$
C_{l}=\bigcup_{n=1}^{n_{l}} C_{l, n}
$$

and define $c_{l}$ to be the common point of $C_{l, 1}$ and $C_{l, n}$. With this construction every $C_{k}, k \geq 2$, is the union of successive wavelike arcs $C_{k, 1}, \ldots$, $C_{k, n_{k}}$ which come from successive line segment parts of $C_{k-1}$. We denote by $h_{k}$ the height and by $d_{k, n}$ the width of the waves of the arc $C_{k, n}$
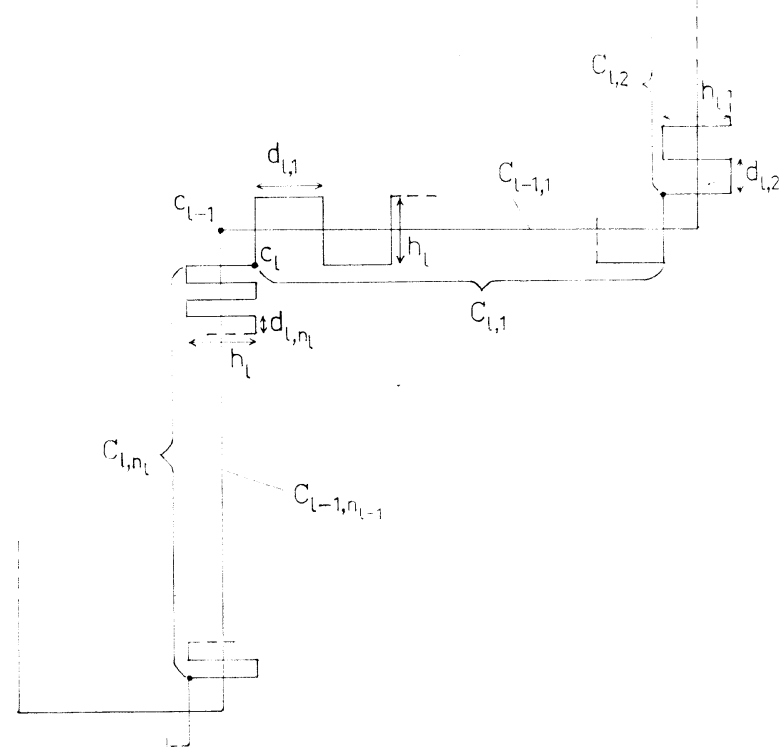

Figure 2. 
(see Fig. 2). In the construction we require now the following additional conditions for $k \geq 2$ :

$$
\begin{gathered}
h_{k}<\min \left\{\frac{d_{k-1, n_{k-1}}}{k n_{k}}, \frac{1}{18}\left(\frac{h_{k-1}}{2 k}\right)^{k}\right\}, \\
d_{k, 1}=h_{k} ; \frac{d_{k, n+p}}{d_{k, n}}<\frac{1}{24}\left(\frac{1}{2 p}\right)^{p} h_{k-1}^{p-1}, p \geq 1, \\
L\left(p, \frac{4 h_{k}}{d_{k, n}}\right)<\frac{h_{k}}{8 d_{k, n+p}}, p \geq 1 .
\end{gathered}
$$

Here $L$ is the function defined by (2.1). It should be noted in (3.1) that $n_{k}$ is determined by $C_{k-1}$.

As a limit of the sequence $C_{1}, C_{2}, \ldots$ we obtain a Jordan curve $\gamma$. We claim that $\gamma$ has the property in the theorem. To prove this suppose there is a $K$-quasiconformal mapping $f: \mathbf{C}^{*} \rightarrow \mathbf{C}^{*}$ such that $f(\gamma)=\gamma$ and such that $f \mid \gamma$ is not the identity. Let $c \in \gamma$ be the limit of the sequence $c_{1}, c_{2}, \ldots$. Because of the assumption, there is a point $z \in \gamma$ such that $z^{\prime}=f(z) \neq z$ and $z, z^{\prime} \neq c$. Suppose that the sequence $c, z^{\prime}, z$ is positively oriented with respect to the bounded complementary domain of $\gamma$. By the Hölder continuity of $f$ there is a constant $M<\infty$ such that $\left|f\left(w_{1}\right)-f\left(w_{2}\right)\right| \leq M\left|w_{1}-w_{2}\right|^{1 / K}$ holds for points $w_{1}, w_{2} \in \gamma$.

We denote by $\gamma_{k, n}$ the subarc of $\gamma$ which comes as a limit from successive deformations of $C_{k, n}$ in the obvious sense. Let now $p>M, K$ be an integer. We choose $\varrho>0$ such that $\overline{B_{\varrho}(z)} \cap \overline{B_{\varrho}\left(z^{\prime}\right)}=\varnothing$ and such that $4 \varrho<\min \left\{|z-c|,\left|z^{\prime}-c\right|,\left|z^{\prime}-f(c)\right|\right\}$. Let $\gamma_{z} \ni z$ be a subarc of $\gamma$ such that $\gamma_{z} \subset B_{\varrho}(z)$ and $\gamma_{z^{\prime}}=f\left(\gamma_{z}\right) \subset B_{\varrho}\left(z^{\prime}\right)$. We can then choose an integer $k>4 L(p, 16)$ such that $d\left(\gamma_{z^{\prime}}\right)>2 h_{k-1}$ and such that there is an integer $n$ such that, if $w \in \gamma_{z}$, then $w \in \gamma_{k, n+p+m}$ for some $m \geq 0$, and if $w^{\prime} \in \gamma_{z^{\prime}}$, then $w^{\prime} \in \gamma_{k, n-m^{\prime}}$ for some $m^{\prime} \geq 0$.

Since $d\left(\gamma_{z^{\prime}}\right)>2 h_{k-1}, \gamma_{z^{\prime}}$ contains a subarc $\gamma_{k, q}$ with $d\left(\gamma_{k, q}\right)>h_{k-1} / 2$. We denote the common points of $C_{k, q}$ and $C_{k-1}$ by $a_{1}, \ldots, a_{r}$ so that the indjces correspond to the successive order on $C_{k, q}$. Let $\tilde{a}_{i}$ be a point on $\gamma_{k, q}$ closest to $a_{i}$ and let $b_{i}$ be a point on $C_{k}$ closest to $\tilde{b}_{i}=f^{-1}\left(\tilde{a}_{i}\right)$, $i=1, \ldots, r$. Then $\left|a_{i}-a_{i+1}\right|=d_{k, q}, i=1, \ldots, r-1$, and $\left|\tilde{a}_{i}-a_{i}\right|$, $\left|\tilde{b}_{i}-b_{i}\right| \leq 2 h_{k+1}, i=1, \ldots, r$.

Suppose that the arc $\alpha=\left(C_{k}-\left\{c_{k}\right\}\right)\left(b_{i}, b_{i+1}\right)$ contains at least 12 successive line segment parts of $C_{k}$ (Fig. 3). Let $e_{0}=b_{i}, e_{1}, \ldots, e_{s-1}$, $e_{s}=b_{i+1}$ be a sequence of successive points on $\alpha$ such that every $\alpha\left(e_{\mu}, e_{\mu+1}\right)$, $\mu=1, \ldots, s-2$, consists of four successive line segment parts of $C_{k}$ and such that $\alpha\left(e_{0}, e_{1}\right)$ and $\alpha\left(e_{s-1}, e_{s}\right)$ do not contain four successive line segment parts of $C_{k}$. Let $\tilde{e}_{\mu} \in \gamma_{z}$ be a point such that $\left|\tilde{e}_{\mu}-e_{\mu}\right| \leq 2 h_{k+1}$ if 


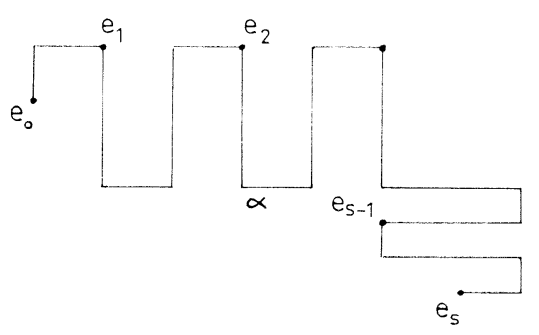

Figure 3.

$\mu=1, \ldots, s-1$, and denote $\tilde{e}_{0}=\tilde{b}_{i}$ and $\tilde{e}_{s}=\tilde{b}_{i+1}$. Then for every $\mu, \mu=0, \ldots, s-1$, there exists an integer $v, v=0, \ldots, s-1,|v-\mu| \leq 2$, such that the following condition holds:

(*) The arc $\beta_{v}=\gamma_{z}\left(\tilde{e}_{v}, \tilde{e}_{v+1}\right)$ has a point $w_{v}$ such that

$$
\frac{\left|\tilde{e}_{v}-w_{v}\right|}{\left|\tilde{e}_{v}-\tilde{e}_{v+1}\right|}>\frac{h_{k}}{4 d_{k, n+p}} .
$$

By applying Lemma 1 to the mapping $f^{-1}$ and the sequence $f\left(w_{v}\right), f\left(\tilde{e}_{v+1}\right)$, $f\left(\tilde{e}_{v}\right), f(c)$ we get by the choice of $\varrho$ the inequality

$$
\frac{\left|\tilde{e}_{v}-w_{v}\right|}{2\left|\tilde{e}_{v}-\tilde{e}_{v+1}\right|} \leq L\left(K, \frac{2\left|f\left(\tilde{e}_{v}\right)-f\left(w_{v}\right)\right|}{\left|f\left(\tilde{e}_{v}\right)-f\left(\tilde{e}_{v+1}\right)\right|}\right) .
$$

If now (*) is satisfied for $v$, we have by the choice of $p$ that

$$
\frac{h_{k}}{8 d_{k, n+p}}<L\left(p, \frac{2\left|f\left(\tilde{e}_{\nu}\right)-f\left(w_{\nu}\right)\right|}{\left|f\left(\tilde{e}_{\nu}\right)-f\left(\tilde{e}_{v+1}\right)\right|}\right) .
$$

The condition (3.3) implies then that

$$
\frac{2\left|f\left(\tilde{e}_{\nu}\right)-f\left(w_{v}\right)\right|}{\left|f\left(\tilde{e}_{v}\right)-f\left(\tilde{e}_{v+1}\right)\right|}>\frac{4 h_{k}}{d_{k, n}} .
$$

But, by the construction of $\gamma$, this is possible only if

$$
d\left(f\left(\beta_{v}\right)\right) \leq 2 h_{k+1} .
$$

If the condition $(*)$ is not satisfied for $v=\mu$, we estimate as follows. Let $v,|v-\mu| \leq 2$, be an integer such that (*) is satisfied for $v$. Then there is a point $\zeta_{\nu} \in \beta_{v}$ such that

$$
\frac{\sup _{w \in \beta_{\nu}}\left|\tilde{e}_{v}-w\right|}{\left|\tilde{e}_{v}-\zeta_{\nu}\right|} \leq 8 .
$$

Again by applying Lemma 1 to the mapping $f$ and the sequence $w, \zeta_{v}$, $\tilde{e}_{v}, c$, where $w \in \beta_{v}$, we get by the choice of $\varrho$ and $p$ the inequality 


$$
\frac{\sup _{w^{\prime} \in f\left(\beta_{v}\right)}\left|f\left(\tilde{e}_{\nu}\right)-w^{\prime}\right|}{\left|f\left(\tilde{e}_{v}\right)-f\left(\zeta_{v}\right)\right|} \leq 2 L(p, 16) .
$$

This together with (3.4) implies

$$
d\left(f\left(\beta_{v}\right)\right) \leq 4 L(p, 16) d\left(f\left(\beta_{v}\right)\right) \leq 8 L(p, 16) h_{k+1},
$$

and since $s<n_{k+1} / 4$, we get thus

$$
\left|\tilde{a}_{i}-\tilde{a}_{i+1}\right|=\left|f\left(\tilde{b}_{i}\right)-f\left(\tilde{b}_{i+1}\right)\right| \leq 2 n_{k+1} L(p, 16) h_{k+1} .
$$

From $n_{k+1} h_{k+1}<d_{k, n_{k}} / k$ and from the choice of $k$ it follows that $\left|\tilde{a}_{i}-\tilde{a}_{i+1}\right|<d_{k, n_{k}} / 2$. But then

$$
\begin{aligned}
\left|a_{i}-a_{i+1}\right| & \leq\left|a_{i}-\tilde{a}_{i}\right|+\left|\tilde{a}_{i}-\tilde{a}_{i+1}\right|+\left|\tilde{a}_{i+1}-a_{i+1}\right|<4 h_{k+1}+d_{k, n_{k}} / 2 \\
& <d_{k, n_{k}} / 2+d_{k, n_{k}} / 2<d_{k, q},
\end{aligned}
$$

which is a contradiction. Hence the arc $\alpha$ contains less than 12 line segment parts of $C_{k}$.

We have now proved that the arc $A=\left(C_{k}-\left\{c_{k}\right\}\right)\left(b_{1}, b_{r}\right)$ contains at most $12(r-1)$ line segment parts of $C_{k}$. Let now $z_{0}=b_{1}, z_{1}, \ldots, z_{\lambda-1}$, $z_{\lambda}=b_{r}$ be a sequence of successive points on $C_{k}$ which divide $A$ into subarcs similarly as the arc $\alpha$ was divided by the sequence $e_{0}, \ldots, e_{s}$ above. Then $\lambda \leq 3 r$. It is now observed that if $z_{\mu}, z_{\mu+1} \in C_{k, t}$ for some $t$ and if $\mu=1, \ldots, \lambda-2$, then $\left|z_{\mu}-z_{u+1}\right|=2 d_{k, t}$. Otherwise we have $\left|z_{\mu}-z_{\mu+1}\right| \leq 2 h_{k}$. Since in any case $d_{k, n+p} / d_{k, n}<1 / 48$, one can conclude that

$$
A \subset \bigcup_{i=0}^{2} C_{k, t-i}
$$

for some $t$. It then follows the estimate

$$
\left|\tilde{b}_{1}-\tilde{b}_{r}\right| \leq 4 h_{k+1}+8 h_{k}+6 r d_{k, n+p}<9 h_{k}+6 r d_{k, n+p} .
$$

By the use of (3.1) and (3.2) we get

$$
\left|\tilde{b}_{1}-\tilde{b}_{r}\right|<\frac{1}{2}\left(\frac{h_{k-1}}{2 k}\right)^{k}+\frac{r}{4}\left(\frac{1}{2 p}\right)^{p} h_{k-1}^{p-1} d_{k, n},
$$

and from $k>4 L(p, 16)>p$ and $r<2 h_{k-1} / d_{k, n}$ the inequality

$$
\left|\tilde{b}_{1}-\tilde{b}_{r}\right|<\left(\frac{h_{k-1}}{2 p}\right)^{p} \text {. }
$$

Combining this with $\left|\tilde{a}_{1}-\tilde{a}_{r}\right|>h_{k-1} / 2$ we are led to the contradiction 


$$
\left|\tilde{a}_{1}-\tilde{a}_{r}\right|=\left|f\left(\tilde{b}_{1}\right)-f\left(\tilde{b}_{r}\right)\right|>p\left|\tilde{b}_{1}-\tilde{b}_{r}\right|^{1 / p}>M\left|\tilde{b}_{1}-\tilde{b}_{r}\right|^{1 / K} .
$$

In the case that the sequence $c, z, z^{\prime}$ is positively oriented with respect to the bounded complementary domain of $\gamma$, the Hölder continuity of $f^{-1}$ is used. The theorem is proved.

University of Helsinki

Helsinki, Finland

\section{References}

[1] Agard, S.: Distortion theorems for quasiconformal mappings. - Ann. Acad. Sci. Fenn. A I 413 (1968).

[2] Ahlfors, L. V.: Quasiconformal reflections. - Acta Math. 109 (1963), 291-301.

[3] Lehto, O. - Virtanen, K. I.: Quasikonforme Abbildungen. - Springer-Verlag, 1965.

[4] Rickman, S.: Characterization of quasiconformal arcs. - Ann. Acad. Sci. Fenn. A I 395 (1966).

[5] -»- Quasiconformally equivalent curves. - To appear in Duke Math. J. 